Research Article

\title{
Interaction Modeling based on Human Behavior Classification
}

\author{
Stefano Filippi \\ DPIA Dept. - University of Udine, via delle scienze 206, Udine, Italy
}

Received 18 October 2019; Accepted 11 January 2020

\begin{abstract}
The variety of human behaviors makes user-product interaction difficult to manage and foresee, especially concerning users running into problems. This research considers several interaction problems and identifies recurring behaviors. Then, it highlights the users, products and environments' aspects corresponding to each of these behaviors. This makes possible foreseeing the behavior of specific users who run into problems while interacting with specific products in specific environments. The results are used to upgrade an existing user-product interaction modeling approach in order to make it able to suggest better-focused product improvements to designers as well as alternative problem solving to different users.
\end{abstract}

Keywords: User-product interaction, Interaction modeling, Human behavior, Functional mock-up for interaction

\section{Introduction}

In recent years, user-product interaction has gained importance mainly because of the increasing product complexity [1-4] and of the need to consider human cognitive activities, personality, etc. [5-6]. Consequently, interaction design $(\mathrm{IxD})$ has become more and more involved in product development processes [7]. The IxD aims at interpreting and modeling user-product dialogues as best as possible [8]. The interpretation highlights relationships between users and products' behaviors during interaction. Modeling products' behaviors is simple; these are always certain and predictable since they are defined at design time. On the contrary, modeling users' behaviors is much more complex; they cannot be known a priori since they depend on the specific aspects of users, products and environments, the three actors of interaction [9-10].

The literature offers several ways to model interaction. Degani, Heymann and Shafto [11] propose a tool that models a correct and reliable interaction between users and products considering products and environments' aspects as well as users' physical ones. Ritter, Kukreja and Amant [12] built user models by exploiting cognitive models to provide a theoretical basis for predicting user behavior. Scarlatos, Tomkiewicz and Courtney [13] generated an agent-based simulation tool where different simulated environments and players interact with each other and with real users. The goal is to teach users how to interpret and behave in case specific economic and social problems happen. Bolton, Bass and Siminiceanu [14] foresee users' mistakes during interaction thanks to specific analytical algorithms and structures. John [15] collected and analyzed behaviors of skilled modelers in order to develop a method, suitable for different design processes and situations, aimed at avoiding novice modelers to make mistakes. As a general consideration, all these works tend to neglect users' subjective aspects like

\footnotetext{
*E-mail address: stefano.filippi@uniud.it

ISSN: 1791-2377 С 2020 School of Science, IHU. All rights reserved.

doi:10.25103/jestr.131.0
}

personality and expectations. On the other side, Paunonen [16] shows that measures of personality can be used to predict a variety of variables useful to represent behaviors in specific social and cultural environments. Komarraju, Karau and Schmeck [17] assert that personality traits can allow predicting college students' academic motivation and achievement. Landers and Lounsbury [18] show that there are strict relationships between personality traits and the way to use Internet. Adali and Golbeck [19] claim that social behaviors could be a strong indicator of user personality. As a general consideration again, all these works seem focused on user personality only; they tend to exclude other, more objective influences like those coming from physical aspects of users, products and environments, from users' expectations, etc.

The research described in this paper aims at deepening the investigation about interaction modeling by analyzing users' behaviors considering objective and subjective influencing aspects together. To get this goal, the research considers several interaction problems and identifies recurring behaviors. Then, it highlights the users, products and environments' aspects corresponding to each of these behaviors. This makes possible foreseeing the behavior of specific users who run into problems while interacting with specific products in specific environments. The results are used to upgrade the existing modeling approach named Functional Mock-Up for interaction (FMUi). To start validating the research outcomes, the interaction between users and a washbasin equipped with automatic faucet and soap dispenser is modelled using the upgraded FMUi; the same interaction is carried on involving real users and the results are compared. The upgraded FMUi can help both researchers in cognitive and behavioral matters and industrial designers. The former can deepen their knowledge about human behavior during problem-solving processes; the latter can have alternative ways to use the products to think about and to tell to their customers, as well as suggestions on how to redesign products in a better-focused way. 
The paper runs as follows. The background section describes what is used here to highlight the recurring behaviors and the current release of the FMUi. The activity section deals with the highlighting of the recurring behaviors, the upgrade of the FMUi and its first validation in the field. The discussion on the research outcomes and some conclusions and hints for future work close the paper.

\section{Background}

\subsection{The Norman's model}

The Norman's model describes the structure of the human actions performed during problem-solving activities [20-22]. This model consists of seven stages. The first two stages set the goals and establish the action needed to achieve these goals. Then, the execution gulf takes place. It represents possible misalignments between the desired action to perform and those the system seems to make available. The third stage selects the action among the available ones and the fourth performs it. The last three stages consist of the perception and interpretation of the system state after the execution of the action. Thanks to them, a decision about the achievement of the goals should spring. A second gulf is present here, the evaluation gulf. This represents possible problems in interpreting the system state and in deciding if the goals have been achieved.

The Norman's model is helpful in this research just because the two gulfs highlight where and when interaction problems could arise.

\subsection{Cognitive architectures}

The cognitive architectures try to reproduce the human mind and its reasoning mechanisms. Since these mechanisms are reputed as the same for every person, the cognitive architectures aim at generating coherent cognition and at modeling the objective component of human behavior [2324]. Many types and examples of cognitive architectures exist, like SOAR [25], ACT-R [23, 26] and CLARION [27]. The cognitive architectures describe human behaviors thanks to six objective characteristics: behaviors are goal-oriented; they take place in complex and detailed environments; they require learning from past experience; they need a large amount of knowledge; they make large use of abstraction; finally, they are flexible, depending on the environment [24].

The cognitive architectures are of great help in this research because of two reasons. First, they provide the six objective characteristics of behavior that will be used complementarily to the subjective aspects of it, managed thanks to the five personality traits described hereafter. Second, they have a synergic role with the Norman's model; while Norman's concerns focus mainly on the "what" happens in every stage of his model, the cognitive architectures go deeply inside the "how" this "what" takes place in the human mind.

\subsection{Human personality}

Human personality is defined as the set of characteristics of a person that account for consistent behavioral patterns over situations and time [28]. Many scientists have tried to underline personality traits to generate a structured and accepted taxonomy of them. Starting from the thirty-five variables highlighted by Cattell [29], other researchers as Tupes and Christal [30], Digman and Inouye [31], and McCrae and Costa [32] developed five common factors to describe the human personality [33]. The big five personality traits is the name of this recognized taxonomy. As described by Rothmann and Coetzer [34], the five traits are as follows.

Extraversion or surgency. It includes traits such as sociability, assertiveness, activity and talkativeness. Extraverts are dynamic and optimistic. Introverts are reserved rather than unfriendly, independent rather than followers. Extraversion implies positive feelings and experiences; therefore, it is considered as a positive affect.

1. Agreeableness. The agreeable person is fundamentally altruistic, sympathetic to others and eager to help them; in return, he/she believes that others will be equally helpful. The disagreeable/antagonistic person is egocentric, skeptical of others' intentions and competitive rather than co-operative.

2. Conscientiousness. It refers to self-control and to the active process of planning, organizing and carrying out tasks. The conscientious person is purposeful, strong-willed and determined. Conscientiousness shows itself in achievement orientation (hardworking and persistent), dependability (responsible and careful) and orderliness (planful and organized). Nevertheless, high conscientiousness may lead to annoying fastidiousness, compulsive neatness or workaholic behavior. Low-conscientious persons may not necessarily lack moral principles, but they are less exacting in applying them.

3. Neuroticism. Neuroticism is a dimension of normal personality indicating the general tendency to experience negative affects such as fear, sadness, embarrassment, anger, guilt and disgust. High neuroticism indicates a person prone to having irrational ideas, being less able to control impulses, and coping poorly with stress. Even higher neuroticism may lead to the risk of some kinds of psychiatric problems. Low neuroticism is indicative of emotional stability. These persons are usually calm, even-tempered, relaxed and able to face stressful situations without becoming upset.

4. Openness to experience or culture. It includes active imagination, aesthetic sensitivity, attentiveness to inner feelings, a preference for variety, intellectual curiosity and independence of judgement. Persons low on openness tend to be conventional in behavior and conservative in outlook. They prefer the familiar to the novel, and their emotional responses are somewhat muted. Persons showing high openness tend to be unconventional, willing to question authority and prepared to entertain new ethical, social and political ideas. Open individuals are curious about both inner and outer worlds, and their lives are experientially richer. They are willing to entertain novel ideas and unconventional values, and they experience both positive and negative emotions more keenly than do closed individuals.

Taking into account human personality is mandatory in this research because it allows dealing with the subjective aspects of human behavior; personality traits help explaining the "why" a specific user behaves in a specific way. This is complementary to the contributions of the Norman's model that focuses on the "what" happens in the human mind to 
perform an action and of the cognitive architectures that describe "how" it happens.

\subsection{Current release of the FMUi}

The FMUi comes from the functional mock-up used to model technological systems [35-37]. The FMUi allows modeling the interaction between a specific user and a specific product in a specific environment during problemsolving activities aimed at satisfying a specific need [38]. During the modeling, the FMUi can highlight possible interaction problems; consequently, the FMUi users interaction designers and/or evaluators - can think about solutions to design/redesign the product in order to prevent those problems from happening again. The FMUi exploitation comes as outlined in figure 1 (this figure will allow catching the contribution of this research at a glance thanks to the comparison with figure 2).

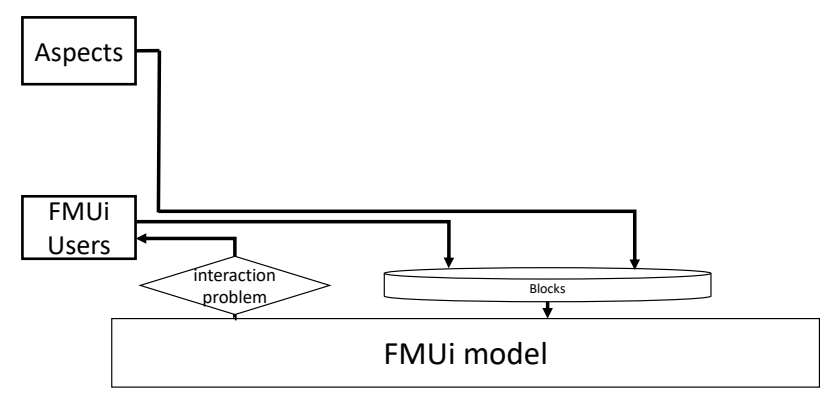

Fig. 1. Outline of the FMUi exploitation.

The FMUi users choose the most suitable blocks to describe user and product behaviors. Each block models a single action made by the user or the product. Inside the blocks, this modeling happens through algebraic equations, Boolean expressions, conditional statements, etc. The interaction model grows up by putting blocks into relationships considering their inputs and outputs. Inputs refer to the three actors of interaction: users, products and environments. Users are described in terms of physical aspects like height and weight, as well as in terms of expectations like desired amounts, temperatures, etc. Products and environments are described only in terms of physical aspects like power, speed, etc., for the former, and room temperature, brightness, etc., for the latter. Outputs represent the results of the simple actions; they consist of physical quantities like temperature and energy, measurements of performance or success flags, all of them expressed in order to allow measurements and comparisons against target values. Success flag values equal to no highlight problems in specific moments of the interaction. As soon as they should show up, the interaction modeling ends.

What follows is an excerpt of the FMUi model describing the interaction of a user with a magnetic card lock system. Two FMUi blocks model the reading of the card and the opening of the lock in case the card code is equal to "1234". The input of the first block is the signal coming from the swipe sensor and the orientation of the card during the swiping action; the output is the success flag about the correct reading of the card and the code read from the card (if swept correctly). The input of the second block is the code read and its output consists in the success flag (positive if the code is equal to the one saved in the system memory; negative otherwise) and the status of the lock (open if the success flag is positive; locked if negative). A negative value of the success flag of the first block could make the FMUi users think about a way to highlight better the correct orientation of the card on the product (using labels, shapes, etc.). If the second block has the success flag equal to no, the FMUi users could think about how to make clearer who is allowed to use the system (to prevent using cards with wrong codes).

This FMUi release allows generating models of different kinds of interactions; nevertheless, although it can highlight if, when and where these interactions could run into problems, it does not give any information/suggestion on how these problems could be overcome. Moreover, failures are always ascribed to the products; users are never considered as responsible for them. As it will be clear in the following, only the involvement of users' personalities (instead of taking common-sense user behavior for granted) and the addition of some facilities can make the FMUi able to model user-product interactions in an exhaustive, effective and constructive way. In fact, these are the requirements for being able to make the user responsible of interaction failures as well and to work on both users and products to improve interactions.

\section{Research activities}

The first research activity analyzes users and products' behaviors when real interactions fail. It exploits the six objective characteristics of human behavior suggested by the cognitive architectures and identifies four recurring behaviors. After that, the reasons why a specific user tries overcoming an interaction problem by engaging one recurring behavior rather than another are investigated. The results are used to upgrade the FMUi. The last research activity exploits the upgraded FMUi in the field to model the interaction of a user with a washbasin equipped with automatic faucet and soap dispenser. In parallel, real users perform the same interaction with a real washbasin. The comparison of the results represents a first validation of the research outcomes.

\subsection{Identifying recurring behaviors ( $R B s)$}

During any interaction, a problem arises when what happens does not match with the user's problem-solving process or, in other words, when there are discrepancies between what the user expects and what happens for real. The execution and evaluation gulfs of the Norman's model represent exactly this (available actions different from the required ones and impossibility to verify the goal achievement) and they are the two moments where discrepancies likely arise. In order to overcome an interaction problem, users can act in several ways or, in other words, they can behave differently. In order to analyze these behaviors, three experts in interaction design and user experience consider different situations. A situation consists of a product expressly set to provoke an interaction problem, a user and an environment. The user interacts with the product in the specific environment, runs into problems for sure and can behaves differently in trying to overcome these problems. The situations to consider are selected by following the suggestions from the representative design method of Dhami, Hertwig and Hoffrage [39]. This method defines a way to conduct experiments where human behavior is involved. If specific requirements are obeyed, the experiments can involve smaller samples of users maintaining the validity and generality of the results. These requirements refer to the presence of stimuli with specific 
characteristics, to the random selection of user samples, to the absence of external interferences during observations and to the observation period. Here, the specific characteristics of the stimuli lead to situations that always involve wellknown products, belonging to different domains. Moreover, every situation refers to the daily life; therefore, the product, the environment and the need to satisfy are common to the majority of the users. Users are selected randomly regarding physical characteristics and personality. The experts do not interfere during the experiments. Finally, the observation period is quite limited because the concern is about the behaviors when the problem occurs; the evolution in the long period is not considered. The satisfaction of the requirements allows limiting the numbers of situations and of the users involved; twelve situations are considered and there are four users for each of them.

The experts describe users' behaviors (how users react to the interaction problems) by assigning values to the six objective characteristics. Behaviors happening in different situations but showing similar values regarding the objective characteristics are generalized and defined as recurring behaviors (RBs). In the end, four RBs are highlighted. Their descriptions appear in the following.

The first RB appeared, among the others, in the opening of a strongbox with a code. The need was to open the strongbox quickly. The user dialed the code but the strongbox did not open. The user tried one more time performing the same actions. The values of the six objective characteristics corresponding to this behavior were as follows. The goal was "open the strongbox" and the environment "a working strongbox". The past experience was "it happened that I dialed the wrong combination of numbers but one more attempt opened the strongbox" and the knowledge was "I know the precise actions to do and the right code to dial". The abstraction was "the product seems to work well, but it does not give the expected results", while the flexibility was "since I am not willing to change my behavior, then I will keep dialing the code". Generally speaking, the past experience shows that the user ran into the same interaction problem in previous situations but, thanks to more attempts, he positively solved the problem. The knowledge highlights that the user knew perfectly the procedures to achieve the goal. The abstraction shows that the environment was perceived as working correctly but the achieved results were not as expected. Finally, the flexibility is very low because the user did not seem to be willing to change his behavior. Overall, similar values of these four objective characteristics showed up in twenty-five situations; therefore, the repetition of the same actions is considered as the first recurring behavior (RB1).

The second RB appeared, among the others, in the closing of a defective window. The need was to close a window in order to avoid catching cold. Since the window was defective, it did not allow to be fully closed. After a first try, the user decided to tolerate a few air flowing in by closing the window as much as possible. The goal was "do not catch cold" and the environment was "room with a defective window and windy weather". The past experience was "it happened that I left the window open without further consequences" and the knowledge was "I know that I am healthy; I am not cold and an air flow will not generate problems to my health". The abstraction was "the product does not work well and the results are not as expected; nevertheless, these results are quite close to the expected ones" and the flexibility was "since I am healthy, then I can adapt myself to the hostile conditions and leave the window slightly open". Generally speaking, the past experience is the willing to accept results different from the expected ones without negative consequences. The knowledge allows considering the effects of results different from the expected ones without drawbacks for the user. The abstraction highlights that the results were not the expected ones, but they were quite close to them. Finally, the flexibility shows that the user was willing to change his expectations. Twenty cases in all showed similar values of these four objective characteristics; therefore, the users' voluntary change of expectations is considered as the second RS (RS2).

The third RB appeared, among the others, in the opening of a mason jar. The need was to access the food in it. After the first unsuccessful attempt, the user warmed up the jar to exploit the thermal strain. In this case, there were changes in the problem-solving process. To overcome the problem, the user preferred to change his strategy instead of trying the same actions or modifying his expectations. The goal was "open a mason jar" and the environment was "a vacuumsealed mason jar with a metal screw top". Regarding the other four characteristics, the past experience was "it happened that when I had to fix a pivot in a hole of a metallic plate, I heated the plate to enlarge the hole" and the knowledge was "I know that metallic materials could expand by heating them". The abstraction was "the product does not let me perform specific actions to achieve the goal" and the flexibility was "since I am willing to try different actions to open the mason jar, then I will use heat to expand the screw top". In this case, the past experience highlights the use of different procedures to solve similar problems belonging to different contexts. The knowledge reports that the user knew alternative procedures to solve problems and that he believed that these procedures could be effective also in different situations. The abstraction highlights the impossibility to perform the desired actions. Finally, the flexibility shows that the user was willing to try alternative actions to achieve the goal. Overall, eighteen situations showed similar values of these objective characteristics; therefore, performing different actions than those planned at the beginning - in other words, changing the problemsolving process - is considered as the third recurring behavior (RB3).

The fourth RB appeared in the situation where room lights should have turned on as soon as the user walked in, since a motion sensor controlled the light system. The interaction of one of the users with the light system failed; the user repeated the same actions (this corresponds to RB1) by exiting and returning in the room more than once but the lights did not turn on anyway. Then the user changed his mind about his/her expectations (RB2) by accepting, just in case, a lower lightness but nothing happened again; finally, making alternative/corrective actions by changing the problem-solving process (RB3) like making noises or walking slower kept the problem unsolved. The only thing to do at this point was to give up about satisfying the need. This happened seven times in all; for this reason, the giving up about satisfying the need is considered as the fourth recurring behavior (RB4).

\subsection{Investigating about the $R B$ selection}

Once the RBs have been identified, the experts analyze the data about users, products and environments collected before. This analysis aims at putting specific users' characteristics like personality, skill, knowledge, past experiences, physical aspects and expectations, as well as products and environments' aspects, into relationships with 
the RBs; in other words, these relationships represent the reasons why a specific user behaves in a specific way to overcome a specific interaction problem in a specific environment. In all, the experts discovered fourteen relationships. These relationships consider users' personality, physical aspects and expectations; the other characteristics (skill, knowledge and past experiences) are neglected for the moment because the analysis did not highlight them enough.

A couple of examples are as follows. One relationship is "IF the user is agreeable, unconscientious and unneurotic, and the faulty product needs more effort than expected in trying to satisfy the need, THEN RB2 must be considered". This was present in the situation of the defective window, where many actions were needed to overcome the damage and the first user, agreeable (willing to accept the conditions of the specific situation without debate), unconscientious (lazy and unwilling to perform too many actions to achieve results) and unneurotic (willing to experience only positive feelings) changed his expectations by tolerating a few air flowing in. Another relationship is "IF the user is conscientious and neurotic, THEN RB3 must be considered". This was present again in the situation with the defective window where another user, conscientious (used to perform even many actions to satisfy needs) and neurotic (prone to experience negative feelings in order to satisfy his needs) changed his problem-solving process to achieve the goal by using an adhesive tape to seal the window to the frame.

\subsection{Upgrading the FMUi}

The next research activity upgrades the FMUi to overcome the limits of the current release as described in Par. 2.4. This comes mainly by adding the module called supervisor. When the modeling activities highlight an interaction problem, this module exploits the four $\mathrm{RBs}$ and the relationships to suggest how to overcome that problem.

The upgraded FMUi maintains the same structure. User personality is added as a new input, expressed through the five traits. This new input is needed to make the model representative for each specific user. The literature offers several methods and tools to measure each trait [40-41]; usually, their values are expressed using a continuous scale as in the Big Five Inventory (BFI) [42]. Here, we consider binary values: the trait itself and its opposite; the involvement of continuous scales is already scheduled as future work. In the upgraded FMUi, if a negative success flag occurs, the modeling activities do not end as it happened before; instead, the supervisor takes the helm and tries to overcome the failure. Input values allow the supervisor to choose the RB that most likely reflects that of the specific user in trying to solve the problem; consequently, the supervisor indicates to the FMUi users how to carry on the modeling activities. In other words, the supervisor suggests how to improve the interaction during the modeling. Figure 2 shows the functioning of the supervisor. The comparison with figure 1 allows catching the differences with the previous release of the FMUi at a glance.

The procedure ruling the functioning of the supervisor works as follows. During modeling activities, when a success flag becomes equal to no, the supervisor considers input values as keywords to highlight the RB that would likely occur thanks to the relationships highlighted in the previous research activity.

If RB1 (repetition of the same actions) is suggested, the supervisor uses the input values to decide how many iterations of the same actions will be likely performed before searching for alternatives. Clearly, RB1 does not involve any consequence from the modeling point of view; therefore, in this case, no suggestions on how to improve interaction are given.

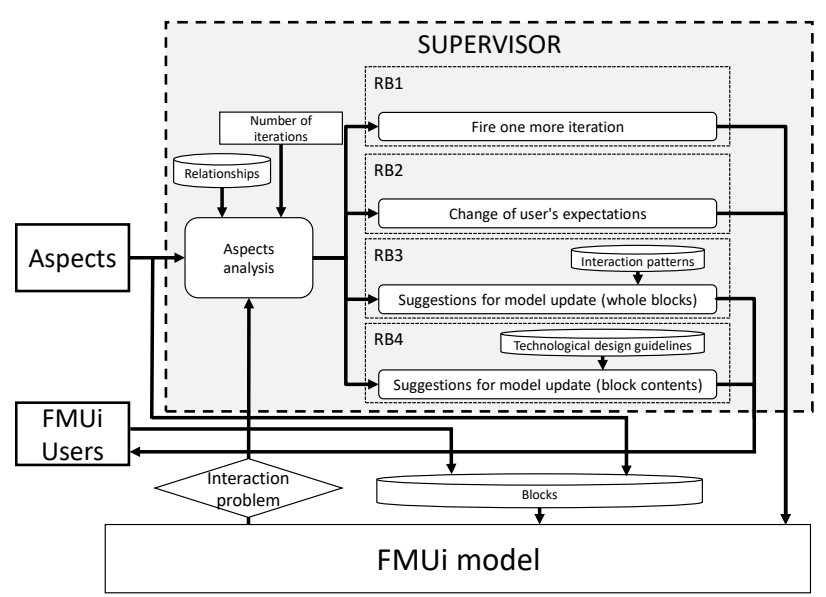

Fig. 2. Functioning of the supervisor.

If RB2 (users' voluntary change of expectations) is suggested, since the supervisor considers inputs (e.g., the user's expectations) as modifiable on the fly, it changes them and these changes allow the modeling activities to be carried on. Although RB2 involves changing input values, also in this case no suggestions on how to improve interaction are proposed (if the user can adapt his/her expectations to the specific situation, there is no need for changes).

If RB3 (change of the problem-solving process) is suggested, the supervisor exploits the FMUi interaction patterns database to suggest an alternative problem solving. An interaction pattern describes a common interaction happening in real situations [43-44]. Patterns are searched through keywords that make the main aspects of the problem to solve explicit. Once the pattern is found, the FMUi users translate it into FMUi blocks and update the model. All of this always refer to problem solving changes; in other words, it can be translated into suggestions for the users on how to change the ways to use that product. On the other side, RB3 never implies product changes. This is intentional, in order to keep RB3 and RB4 clearly apart from each other to make their management easier.

The selection of RB4 (giving up about need satisfaction) comes when interaction goes towards an unsuccessful end because all the other RBs are not applicable. To avoid this, the supervisor tries to solve the problem by suggesting modifications to the product. The technological design guidelines database is exploited here. It consists of wellknown guidelines like those for embodiment design [45] and the 40 principles of the TRIZ theory [46]. Thanks to the similarities in the structures of the different sets of technological design guidelines, their keyword-based search is performed as it happens for the interaction patterns. Once selected, the guidelines allow the content of blocks already present in the FMUi model and representing the product behavior to be modified by the FMUi users in order to let the product go along with the users' problem-solving process as best as possible. RB4 implies certain repercussions from the IxD point of view since the FMUi users must rethink the product according to the suggestions coming from the exploitation of the technological design guidelines.

Finally, if the value of a success flag remains equal to no even after the exploitation of all the suitable RBs, the 
supervisor must give up and the interaction comes inevitably to an unsuccessful end. In this case, the FMUi cannot be of further help in improving the interaction.

It is worth to say that the FMUi requires a reasonable number of runs involving different users before to trust its outcomes and modify products or problem-solving activities accordingly.

\subsection{FMUi exploitation}

This section describes the FMUi exploitation in generating the model of a whole interaction. This shows the capabilities of the upgraded FMUi in suggesting how to foresee, overcome and avoid interaction problems.

The interaction considered here is that of the user John who wants to wash his hands using a washbasin equipped with a faucet releasing water thanks to a photocell and with an automatic soap dispenser. John is $1.6 \mathrm{~m}$ tall and, recently, he burned one hand while cooking. John is a dynamic person; he does many activities every day; therefore, he is considered as extravert. Moreover, he does not take care of secondary matters not directly related to the hand washing or to the burn as the soap quantity and the time spent to get the result; so, he is agreeable. John is very demanding about washing his hands (at lunchtime, as soon as he feels them dirty, etc.) because he is a health fanatic; therefore, John is reputed as conscientious. Finally, the burn made John requiring washing his hands with a precise water temperature that he experienced to be the best one. This makes consider John as neurotic about searching for this temperature and closed to experience; he is scared of using different temperatures than the expected ones because the burn could get worse.

The interaction consists in three separate moments: the first moment regards the actions to make the water flow from the faucet; the second deals with the water temperature setting; the third concerns the achievement of the soap to wash the hands.

\section{First interaction moment: make the water flow}

The FMUi users start generating this part of the model by introducing and connecting the blocks representing two simple behaviors: the hands positioning (block B1) and the hands distance evaluation and water release (B2). Block B1 models the approach of the hands to the faucet. The outcome of this block (hands distance) depends on the user's height (input) and on the dimensions of the washbasin (known in the model because defined at washbasin design time). Block B2, describing the behavior of the faucet, compares the hands distance against the required one (equal to $10 \mathrm{~cm}$ as set at faucet design time) and manages the release of the water. The water will flow only if the hands are close enough to the faucet (distance less than or equal to $10 \mathrm{~cm}$ ). If this happens, the water flows from the faucet and the success flag is set to yes; otherwise, there is no water flowing and the success flag is set to no.

Considering John, when he approaches the faucet and tries placing his hands near it (as modeled by B1), B2 evaluates the hands distance as too big for the water to be released and this is due to the fact that John is quite short, although he is very close to the washbasin; therefore, the interaction fails (what happens does not match the user's expectations) and the success flag of B2 is set to no. This activates the supervisor that, by exploiting the relationships, suggests that John will likely repeat the same actions to satisfy his need because he is extravert and conscientious. Therefore, RB1 is selected; the maximum number of John's attempts to make the water flowing before to do something else is set as four. Then a new iteration, representing the second attempt to make the water flowing, is fired. This attempt fails as well because John's hands are still too far and a new iteration takes place. Unfortunately, the same result happens for the third and fourth attempts. The maximum number of iterations is reached and the success flag of B2 is still equal to no. Therefore, the supervisor exploits again the relationships to highlight the next $\mathrm{RB}$ to consider, excluding the relationships leading to RB1 because the maximum number of iterations of the same actions has been reached. To John, it is imperative to get the hands washed because he is conscientious; therefore, RB3 is suggested as the best choice, since RB2 or RB4 cannot apply. RB2 would imply John accepting a different result from the expected one (no water instead of flowing water), but this would keep the hands dirty and John cannot bear this because of his conscientiousness; for the same reasons, also RB4, where John would give up about washing his hands, is not suitable. Due to the RB3 selection, the interaction patterns are searched for a solution to solve the problem consisting in the low user's height and this is used as a search keyword. The suggested solution involves a footstool. Two new blocks describing the simple user behaviors of positioning the footstool close to the washbasin (B2') and of stepping on it (B2") are added to the model by the FMUi users. The use of the footstool increases John's height, the input of B1. Thanks to the new value of the user height, B2 lets the water flow and this corresponds to the success of this first interaction moment. Figure 3 shows the FMUi model after RB1 (three times) and RB3.

Considering the implications of these modeling activities on the IxD, RB1 does not provide any suggestion because it consists in the repetition of the same actions, in the same way, with the same product. On the contrary, RB3 suggests an alternative problem-solving process thanks to the exploitation of the footstool.

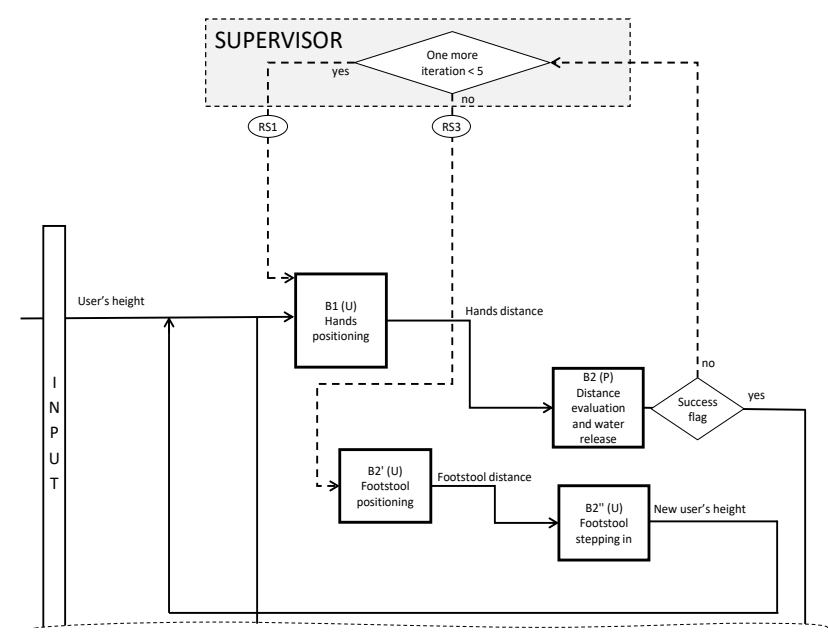

Fig. 3. Part of the FMUi model corresponding to the first interaction moment.

\section{Second interaction moment: water temperature setting}

The FMUi users consider three blocks to model this moment. Block B3 describes the simple behavior of the faucet that sets the temperature of the water respect to the current position of the regulatory knob. The input consists of the success flag coming from B2 and of the modification of the knob position (if any). Block B4 describes the user's evaluation of the water temperature. The input consists of 
the water temperature coming from B3 and of the user's expected one. This can have four values (expressed using the user's language): "cold" (less than $\left.20^{\circ} \mathrm{C}\right)$, "warm" $\left(20^{\circ} \mathrm{C}\right.$ to $29^{\circ} \mathrm{C}$ ), "hot" $\left(30^{\circ} \mathrm{C}\right.$ to $39^{\circ} \mathrm{C}$ ) and "scalding" (more than $40^{\circ} \mathrm{C}$ ). The output consists of the success flag about the user's evaluation - it is set to yes if the current water temperature matches the expected one, no otherwise - and of the water temperature due to the knob position. Block B5 models the simple behavior of the user in adjusting the water temperature by turning the knob. The output consists of the modification of the knob position. This output is loopedback to B3 for a new setting of the water temperature performed by the faucet.

Considering John's interaction, it starts with the faucet that sets the water temperature according to the current position of the knob (supposed as positioned in the middle of its range). After that, John evaluates this temperature. Unfortunately, his expectations are not satisfied because the water is too cold. The success flag of B4 is set to no and the supervisor is involved. The relationships are exploited and RB3 (change the problem-solving process) is suggested as the recurring behavior to consider. In fact, John does not likely repeat the same actions (RB1) because the temperature would be the same and he cannot bear this because he is neurotic and, on the other side, John does not modify his expectations (RB2) because of his closeness to experience; moreover, he cannot give up about washing his hands (RB4) due to his conscientiousness. RB3 (represented in figure 4 using a single-dashed arrow) requires searching interaction patterns for a solution to solve the problem of the wrong water temperature. This suggests the pattern "search for further/hidden product functionalities"; consequently, the FMUi users look at the faucet and decide to make John perceiving and exploiting the existing adjustment system (the knob). Therefore, block B5 is introduced in the model. John's characteristics suggest that he would turn the knob just one notch because he is conscientious and closed to experience; therefore, he is worried about keeping experiencing wrong temperatures coming from wider changes. The faucet sets the new temperature accordingly and John evaluates it. Again, the water seems too cold. The success flag of B4 is set again to no; this is represented in Figure 4 using a double-dashed arrow. Due to the possibility of modifying water temperature introduced by the previous modeling activity, the relationships, suggest RB1 as the behavior that would likely occur. John is extravert; so, he is reputed to try the adjustment at most four times. John moves the knob one more notch (block B5) but the water remains too cold. The third iteration lets the water too cold as well while the fourth one makes it too hot. Now, RB1 cannot happen again because the maximum number of iterations has been reached; moreover, as said before, John is not willing to modify his expectations (RB2); at the same time, he cannot change anything in the problem-solving process (RB3) because the faucet offers only the knob to control the water temperature and it did not allow John to reach the expected temperature. Therefore, RB4 is suggested, corresponding to giving up in satisfying the need unless some changes to the product are introduced. The technological design guidelines are searched using "variable setting" as keyword. The best solution consists in allowing selecting values closer to each other in order to perform finer tunings. This suggestion comes from the first principle of the TRIZ theory "segmentation; increase the degree of fragmentation or segmentation". Changing the knob sensitivity would result in adding new possible positions to the knob; consequently, intermediate values for the water temperature would be available. This product modification would leave the user behavior as it is but, thanks to this modification, the user could find the expected temperature because of the higher flexibility offered by the knob. From the modeling point of view, the FMUi users modify the content of B5 to reflect the narrower gap between temperature values corresponding to two consecutive notches. In Figure 4, the modified block B5 is labeled as $\mathrm{B}^{*}$ and the two blocks are represented in different ways. Now, John applies one more notch turn to the knob; this time he achieves an intermediate value that was not available before the modification of B5, due to the coarser adjustment. The faucet sets a new water temperature and John evaluates it. This temperature corresponds to the expected one and the success flag is set to yes. Figure 4 shows the FMUi model of the second interaction moment, reflecting the effects of RB3, RB1 (three times) and RB4.

The implications of these modeling activities on the IxD are as follows. RB3 suggests a different problem solving consisting in an alternative use of the product (the use of the knob to adjust the temperature). RB1 does not provide any suggestion because it consists in the repetition of the same actions, in the same way and with the same product. Finally, RB4 leads to the development of a more sensitive regulatory system for the water temperature.

\section{Third interaction moment: achievement of the soap}

The third moment consists of three simple behaviors: the hand positioning, the soap release and the user's evaluation of the quantity of it. These are described by blocks B6, B7 and B8, respectively. B6 has the same content as B1 because both of them aim at managing the user's hand positioning. The input consists of the user's height and of the success flag of B4. The output is the hand distance from the dispenser. In $\mathrm{B} 7$, the dispenser evaluates the hand distance in order to decide if to release the soap or not. The input of B7 is the hand distance and the output is the success flag and, eventually, the quantity of soap released. If the hand distance is correct, the dispenser gives the specific quantity of soap and the success flag is set to yes. Finally, B8 models the user's evaluation of the soap quantity. The input consists of the quantity of soap released by the dispenser and of the success flag of B7, together with the user's expected quantity of soap. The output is the final success flag representing the success of the whole interaction, since with the water flawing at the expected temperature and with the expected quantity of soap, the user can wash his/her hands.

Considering now John's interaction, the FMUi users introduce $\mathrm{B} 6$ to represent the definition of the hand distance. The height of John is computed considering the use of the footstool and B6 sets the same hand position as B1. B7 evaluates the hand position as inside the distance range to allow the soap to be released; therefore, the success flag of B7 is set to yes. This value enables the release of a predefined quantity of soap and, in turn, it allows the addition of block B8 representing John's evaluation of the soap quantity. The quantity of soap released by the dispenser appears as scarce, compared to John's expected one. Therefore, the success flag value of B8 is set to no. Now, the relationships suggest $\mathrm{RB} 2$ as the recurring behavior that would likely occur because John is agreeable on secondary matters as, indeed, the quantity of soap. Therefore, the value of the input representing the expected quantity of soap is changed on the fly from "a handful" to "some drops" (John reputes this quantity a reasonable trade-off to get the result). 
Consequently, the success flag of B8 is set to yes and the interaction comes to the successful end; the water flows at the expected temperature, the soap quantity is satisfactory and John can wash his hands. Figure 5 shows the third part of the FMUi model, where RB2 is considered.

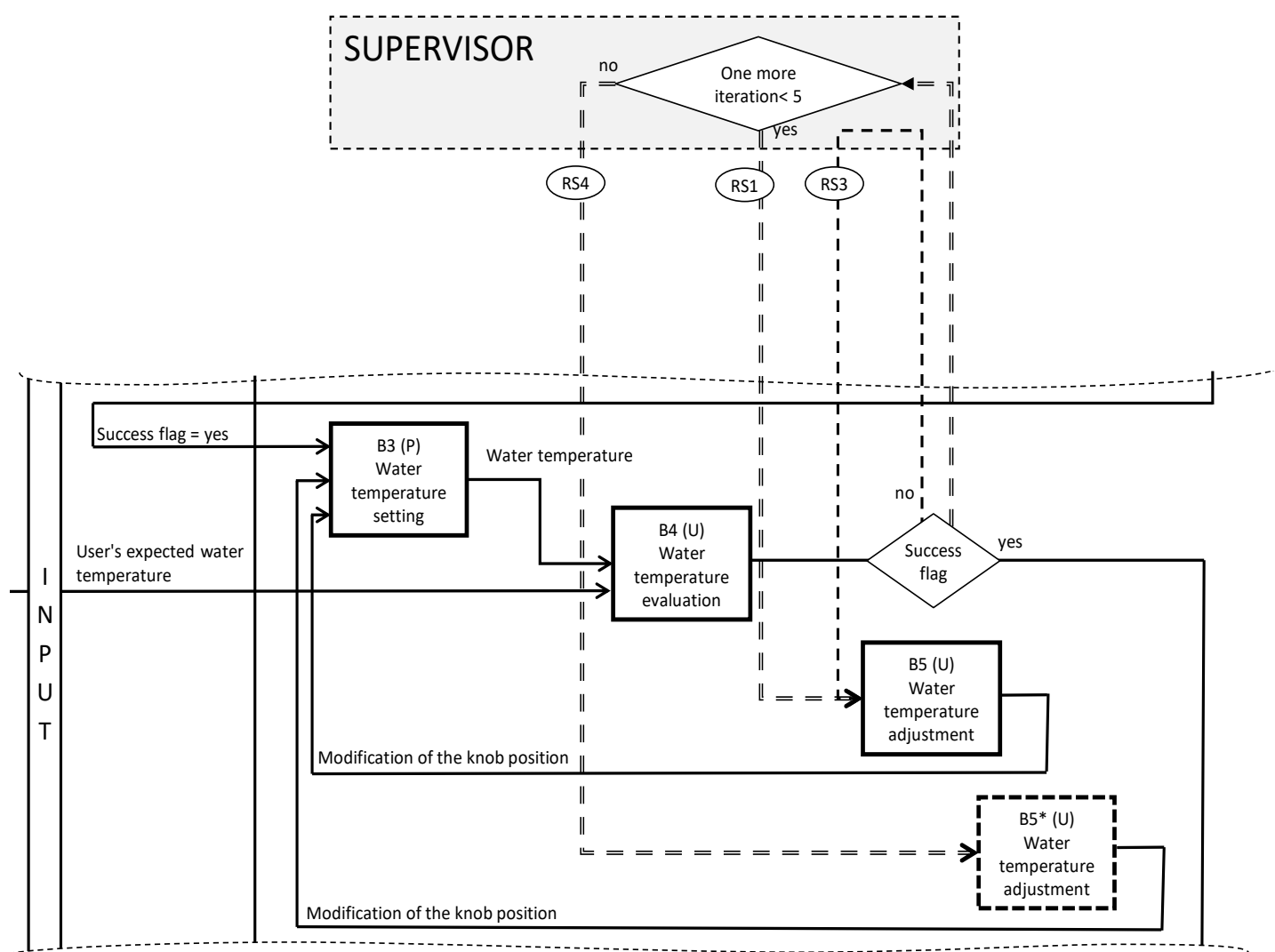

Fig. 4. Part of the FMUi model corresponding to the second interaction moment.

There are no implications of these modeling activities on the IxD because RB2 represents the users' voluntary change of expectations and this does not require any changes to the problem solving or to the product.

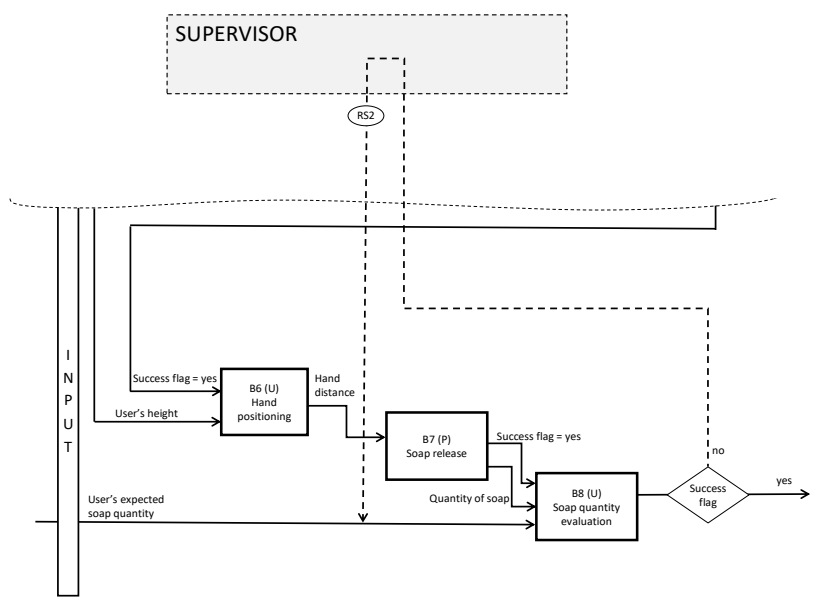

Fig. 5. Part of the FMUi model corresponding to the third interaction moment.

In summary, the FMUi exploitation in modeling the interaction of the specific user John with the washbasin suggests two interaction improvements. The first one, coming from RB3 in the first interaction moment, consists in changing the problem-solving process by involving a footstool to increase John' height. The second one, coming from RB4 in the second moment of interaction, refers to the design of a more sensitive system for the regulation of the water temperature.

\subsection{First validation of the upgraded FMUi}

In order to start validating the accuracy of the models generated using the upgraded FMUi in representing real interactions, tests are conducted with real users performing the same interaction described in Sect. 3.4. At the end of these tests, real users and products' behaviors are compared with those described by the FMUi model.

The real interaction happens in a bathroom with a washbasin equipped with automatic faucet and soap dispenser. On the floor, in a corner of the bathroom, there is a footstool. Two interaction experts follow the tests and compare behaviors afterward. The selection of the users happens thanks to an online questionnaire aimed at highlighting personality traits and specific physical aspects (height, possible temporary or permanent disablements, etc.). The questionnaire is sent to thirty people; in the end, four users are selected because of their similarities to John's characteristics.

At the beginning, the experts introduce the test, need included. After that, the four users (from now on, U1 to U4) start interacting with the washbasin, one at a time to avoid bias, without any interruption or help from the experts except for those situations where users are going to give up because they cannot satisfy the need. The experts observe carefully the interactions and collect data throughout. Once the last interaction comes to the end, the experts start analyzing the data to compare behaviors.

Considering the first interaction moment, all the users approached the faucet with their hands. For all of them, the water did not flow (exactly as John). Then, U1 and U3 after three unsuccessful attempts, U2 after four (as John) and U4 after two, tried to do something else. All of them used the 
footstool. U1 and U2 had immediate success (as John); U3 and $\mathrm{U} 4$ positioned the footstool wrongly and failed to get the water flowing; nevertheless, it was enough to reposition the footstool to have success as well. The differences between the modeled and real behaviors consist in the number of attempts made before to change something and in the correct positioning of the footstool. The different number of attempts could depend on the fact that users could not necessarily be as extravert as John; consequently, they could be less dynamic and repeat the same actions less times than he repeats. As said in 3.2, the FMUi can assign just two values to every personality trait, the trait itself and its opposite; different nuances of extraversion cannot be managed now. The differences in the correct positioning of the footstool could depend on the user skill in evaluating the mutual positions of objects and their distance. User skills are not considered in the FMUi yet.

Considering the second interaction moment, U1, U3 and U4 tried setting the water temperature three times and U2 four (as John), all of them with no success. When U1 and U2 gave up (as John) because it was unbearable for them washing the hands at a wrong water temperature, the experts intervened by changing the faucet setup to allow the finer regulation. Thanks to this, the two users got the correct temperature, both of them after two attempts (John was able to set it at the first attempt). On the contrary, U3 and U4, at the end of their attempts, accepted the water temperature as it was, although it did not match their expectations. The differences between the modeled and real behaviors consist in the number of attempts to adjust the water temperature with the original regulatory system, in accepting different values of the water temperature than the expected ones (vs. giving up) and in the number of attempts to achieve the expected water temperature with the finer regulation. The different number of attempts to adjust the water temperature with the original regulatory system could depend again on the different extraversion degree than John, as it happened in the first interaction moment. As already said, the FMUi cannot manage the "less extravert than" value. The differences about accepting different values of the water temperature than the expected ones (vs. giving up) could depend on the fact that U3 and U4 had no burns on their hands. Therefore, after some attempts, they accepted the temperature quite similar to the expected one. No users with burns answered to the questionnaire; therefore, some differences in the behaviors depending on this were expected. Finally, the different number of attempts to achieve the expected water temperature using the finer regulation could depend on the different value of conscientiousness of the users; again, the FMUi cannot consider such nuances now.

Considering the third interaction moment, U1 and U3 behaved exactly as John; they approached the dispenser just once and accepted the soap quantity; on the contrary, U2 and U4 tried to achieve a higher quantity of soap by repeating the same actions with the dispenser. The differences between the modeled and real behaviors focus again on the number of attempts. U2 and U4 were less agreeable than John on secondary matters was; therefore, they repeated the same actions to achieve a higher quantity of soap. As already said, the five personality traits in the FMUi can take just two values; therefore, the FMUi cannot manage the "less agreeable than" value.

In summary, the differences between the modeled and real behaviors consist of the number of attempts before to do something else, of the correct use of other products and of accepting different values than the expected ones vs. giving up.

\section{Discussion}

The first validation highlighted a good connection between the model generated by the upgraded FMUi and what happened for real. The behaviors of real users were very similar to those foreseen by the model. The users, products and environments' aspects seem considered and managed correctly and effectively, although the characterization of users' personalities should be deepened, as demonstrated by the missing full correspondence. The successful FMUi exploitation in generating the model of a whole interaction also starts witnessing the FMUi usability by non-developers.

Nevertheless, some research drawbacks must be highlighted as well. Considering the current level of the FMUi development, blocks and relationships are quite numerous; nevertheless, they cannot cover every possible interaction. Sometimes, the FMUi users must generate missing blocks by themselves on the fly or suggest which $\mathrm{RB}$ to select if the supervisor does not suggest suitable relationships. This requires skill and knowledge about human behavior and cognition, as well as about the context where interactions take place. The amount of interaction patterns is quite low and they are not structured to be searched using keywords easily. Sometimes FMUi users must select patterns by themselves following the suggestions of the supervisor. The same counts for the technological design guidelines, where the FMUi users must translate the guidelines into modifications of existing blocks by themselves. Another drawback consists of the capability of modeling only one RB at a time. Situations like that of a user who is willing to change his problem-solving process (RB3) but the best way to put this into practice would be changing the product behavior (RB4) cannot be managed now. In fact, the suggestions coming from RB3 propose just changes to the problem-solving activities that cannot have repercussions on the design/redesign of the product. The last drawback regards the user personality. Now, every trait can take just two values and has the same weight. Moreover, the traits are considered separated from each other; nevertheless, in real life, they have different nuances and weights and, of course, influence each other.

\section{Conclusions}

The research described in this paper aimed at modeling userproduct interactions as best as possible in order to get suggestions on how to improve them, all of this based on a classification of human behaviors. This was achieved with the definition of four recurring behaviors happening when interactions run into problems. Then, the existing approach for interaction modeling named functional mock-up for interaction (FMUi) has been upgraded to make it able to manage these recurring behaviors. An example of FMUi exploitation has been proposed to show the FMUi capabilities in suggesting alternative uses of products or modifications of them. A first validation of the FMUi has been proposed as well. Both researchers in cognitive and behavioral matters and industrial designers seem entitled to exploit this new release of the FMUi. Researchers could deepen their knowledge about human behavior by applying the FMUi and comparing the resulting models with real 
user-product interactions. This would allow highlighting the aspects that influence users' reasoning and decision making and the different values these aspects could assume representing different behavior nuances. Industrial designers could be helped in foreseeing user behaviors in specific situations, thus reducing the number of real users to involve in tests or avoiding tests at all. Moreover, they can have alternative ways to use the products to think about and to tell to their customers as well as suggestions on how to redesign products.

Some research perspectives are as follows. Some facilities to generate blocks and highlight further relationships need to be introduced, also by exploiting unconventional tools and methods like recommender systems [47], etc. All of this will allow the FMUi users to build models without specific knowledge in cognitive matters or about the context where the interactions take place. Interaction patterns and technological guidelines databases must be improved, both from the organization and population points of view, by adding more principles and standards related to interaction issues and facilities to apply structural changes to existing blocks, respectively. Selecting and modeling more than one $\mathrm{RB}$ at a time must be accounted for because there are situations where the RBs are related each other. Their inter-relationships could actually be fundamental to solve complex problems. Finally, the aspects describing user personality need to be further investigated by taking into account more values than they allow now (trait itself or its opposite only) because small variations could lead to big differences in users' behaviors. Moreover, these aspects must have different weights depending on the need, the specific situation and product, etc.

This is an Open Access article distributed under the terms of the Creative Commons Attribution License

\section{References}

1. B. Shackel, "Usability - Context, framework, definition, design and evaluation", Interacting with Computers, 21(5-6), pp. 339-346 (2009).

2. L.E. Janlert and E. Stolterman, "Complex interaction", ACM Transactions on Computer-Human Interaction, 17(2), pp. 8.1-8.32 (2010).

3. M. Hertzum and T. Clemmensen, "How do usability professionals construe usability?", International Journal of Human-Computer Studies, 70(1), pp. 26-42 (2012).

4. S. Filippi and D. Barattin, "X for design: A usable framework for enhancing design activities", Journal of Engineering Science and Technology Review, 12(4), pp. 183-194 (2019).

5. J. Park, S.H. Han, H.K. Kim, Y. Cho and W. Park, "Developing Elements of User Experience for Mobile Phones and Services: Survey, Interview, and Observation Approaches", Human Factors and Ergonomics in Manufacturing \& Service Industries, 23 (4), pp. 279-293 (2013).

6. S. Valtolina, B.R. Barricelli, M. Mesiti and M. Ribaudo, "UserCentered Design of E-Learning Tools for Users with Special Needs: The VisualPedia Case Study", Interaction Design and Architecture(s) Journal, 13-14, pp. 47-55 (2012).

7. M. Barajas and B. Agard, "Selection of products based on customer preferences applying fuzzy logic", International Journal on Interactive Design and Manufacturing, 5(4), pp. 235-242 (2011).

8. R. Heimgärtner, "Cultural Differences in Human-Computer Interaction: Towards Culturally Adaptive Human-Machine Interaction", Walter de Gruyter Munich (2012).

9. S. Fuchs, J. Schwarz and F.O. Flemisch, "Two steps back for one step forward: revisiting augmented cognition principles from a perspective of social system theory", in Proceedings of the 16th International Conference on Human-Computer Interaction, Lecture Notes in Computer Science book series LNCS, 8534, pp.114-124. Springer (2014).

10. E.H. Calvillo-Gámez, P. Cairns and A.L. Cox, "Assessing the Core Elements of the Gaming Experience", Chapter four in Evaluating User Experience in Games, pp. 47-72, Springer, London (2010).

11. A. Degani, M. Heymann and M. Shafto, "Modeling and formal analysis of human-machine interaction", in The Oxford Handbook of Cognitive Engineering, pp. 433-448, Oxford University Press, New York (2013)

12. F.E. Ritter, U. Kukreja and R.S. Amant, "Including a Model of Visual Processing with a Cognitive Architecture to Model a Simple Teleoperation Task", Journal of Cognitive Engineering and Decision Making, 1(2), pp. 121-147 (2007).

13. L.L. Scarlatos, M. Tomkiewicz and R. Courtney, "Using an AgentBased Modeling Simulation and Game to Teach Socio-Scientific Topics", Interaction Design and Architecture(s) Journal, 19, pp. 7790 (2013).

14. M.L. Bolton, E.J. Bass and R.I. Siminiceanu, "Generating phenotypical erroneous human behaviour to evaluate human- automation interaction using model checking", International Journal of Human-Computer Studies, 70(11), pp. 888-906 (2012).

15. B.E. John, "Reducing the Variability between Novice Modelers: Results of a Tool for Human Performance Modeling Produced through Human-Centered Design", in Proceedings of the 19th Conference on Behavior Representation in Modeling and Simulation, pp. 95-102 (2010).

16. S.V. Paunonen, "Big Five Factors of Personality and Replicated Predictions of Behavior", Journal of Personality and Social Psychology, 84 (2), pp. 411-424 (2003).

17. M. Komarraju, S.J. Karau and R.R. Schmeck, "Role of the Big Five personality traits in predicting college students' academic motivation and achievement", Learning and Individual Differences, 19, pp. 47-52 (2009).

18. R.N. Landers and J.W. Lounsbury, "An investigation of Big Five and narrow personality traits in relation to Internet usage", Computers in Human Behavior, 22, pp. 283-293 (2006).

19. S. Adali and J. Golbeck, "Predicting Personality with Social Behavior", in Proceedings of the International Conference on Advances in Social Networks Analysis and Mining (ASONAM). IEEE (2012)

20. D. Norman, "The Design of Everyday Things: Revised and Expanded Edition", Basic Books, New York (2013).

21. D.A. Norman and S.W. Draper, "User Centered System Design; New Perspectives on Human-Computer Interaction", Erlbaum Associates Inc. Hillsdale, New York (1986).

22. A.A.A. Ibrahim and A. Hunt, "An HCI Model for Usability of Sonification Applications", Lecture Notes in Computer Science, 4385, pp. 245-258 (2007).

23. J.R. Anderson, D. Bothell, M.D. Byrne, S. Douglass, C. Lebiere and Y. Qin, "An integrated theory of the mind", Psychological Review, 111(4), pp. 1036-1060 (2004).

24. J.F. Lehman, J. Laird and P. Rosenbloom, "A gentle introduction to Soar, an architecture for human cognition", 2006 update (2006). https://web.eecs.umich.edu/ soar/sitemaker/docs/misc/GentleIntrod uction-2006.pdf

25. J.E. Laird, "Extending the Soar cognitive architecture", in Proceedings of the Conference on Artificial General Intelligence 2008, pp. 224-235. IOS Press, Amsterdam (2008).

26. R.L. West and S. Pronovost, "Modeling SGOMS in ACT-R: Linking Macro- and Microcognition", Journal of Cognitive Engineering and Decision Making, 3(2), pp. 194-207 (2009).

27. I. Naveh and R. Sun, "A cognitively based simulation of academic science", Computational \& Mathematical Organization Theory, 12(4), pp. 313-337 (2006).

28. S. Karimi and M.R. Kangavari, "A Computational Model of Personality", Procedia - Social and Behavioral Sciences, 32, pp. 184-196 (2012).

29. R.B. Cattell, "The description of personality: Basic traits resolved into clusters", The Journal of Abnormal and Social Psychology, 38(4), pp. 476-506 (1943). 
30. E.C. Tupes and R.E. Christal, "Recurrent personality factors based on trait ratings", Technical Report No. ASD-TR-61-97, Lackland Air Force Base, TX. U.S. Air Force (1961).

31. J.M. Digman and J. Inouye, "Further specification of the five robust factors of personality", Journal of Personality and Social Psychology, 50, pp. 116-123 (1986).

32. R.R. McCrae and P.T. Costa, "Validation of the five-factor model of personality across instruments and observers", Journal of Personality and Social Psychology, 52(1), pp. 81-90 (1987).

33. L.R. Goldberg, "An Alternative 'Description of Personality': The Big-Five Factor Structure", Journal of Personality and Social Psychology, 59(6), pp. 1216-1229 (1990).

34. S. Rothmann and E.P. Coetzer, "The big five personality dimensions and job performance", Journal of Industrial Psychology, 29(1), pp. 68-74 (2003).

35. F. Zorriassatine, C. Wykes, R. Parkin and N. Gindy, "A survey of virtual prototyping techniques for mechanical product development", in Proceedings of the Institution of Mechanical Engineers, Part B: Journal of Engineering Manufacture, 217, pp. 513-530 (2003).

36. F. Ferrise, M. Bordegoni and U. Cugini, "Interactive Virtual Prototypes for testing the interaction with new products", Computer-Aided Design and Applications, 10, pp. 515-525 (2013).

37. O. Enge-Rosenblatt, C. Clauß., A. Schneider and P. Schneider, "Functional Digital Mock-up and the Functional Mock-up Interface - Two Complementary Approaches for a Comprehensive Investigation of Heterogeneous Systems", in Proceedings of the 8th Modelica Conference. Linköping University Electronic Press, Linköpings (2011).

38. S. Filippi, D. Barattin, F. Ferrise, M. Bordegoni and U. Cugini, "Human in the loop: a model to integrate interaction issues in complex simulations", in Proceedings of the 15th International
Conference on Human-Computer Interaction, HCI2013, pp. 242251. Springer (2013).

39. M.K. Dhami, R. Hertwig and U. Hoffrage, "The role of Representative Design in an ecological approach to cognition", Psychological Bulletin, 130(6), pp. 959-988 (2004).

40. R.R. McCrae, P.T. Costa and T.A. Martin, "The NEO-PI-3: A More Readable Revised NEO Personality Inventory", Journal of Personality Assessment, 84(3), pp. 261-270 (2005).

41. L.R. Goldberg, J.A. Johnson, H.W. Eber, R. Hogan, M.C. Ashton, C.R. Cloninger and H.G. Gough, "The international personality item pool and the future of public-domain personality measures", Journal of Research in Personality, 40(1), pp. $84-96$ (2006).

42. C.J. Soto and O.P. John, "Ten facet scales for the Big Five Inventory: convergence with NEO PI-R facets, self-peer agreement, and discriminant validity", Journal of Research in Personality, 43, pp. 84-90 (2009).

43. A. Gangemi, "Ontology design patterns for semantic web content", Lecture Notes in Computer Science, 3729, pp. 262-276 (2005).

44. C. Hubscher, S.L. Pauwels, S.P. Roth, J.A. Bargas-Avila and K. Opwis, "The organization of interaction design pattern languages alongside the design process", Interacting with Computers, 23(3), pp. 189-201 (2011).

45. G. Pahl and W. Beitz, "Engineering Design: A Systematic Approach", Springer Science \& Business Media, London (2013).

46. G. Altshuller, L. Shulyak and S. Rodman, "40 Principles: TRIZ Keys to Innovation", Technical Innovation Center, Inc. Worcester (2002).

47. S.S. Sohail, J. Siddiqui and R. Ali, "Classifications of recommender systems: A review", Journal of Engineering Science and Technology Review, 10(4), pp. 132-153 (2017). 